EPJ Web of Conferences 32, 02018 (2012)

DOI: $10.1051 /$ epjconf/20123202018

(C) Owned by the authors, published by EDP Sciences, 2012

\title{
In vessel characterization and first power tests on plasma of the Real-Time controllable EC launcher on FTU Tokamak
}

\author{
A. Moro ${ }^{1, a}$, E. Alessi ${ }^{1}$, G. Artaserse ${ }^{2}$, W. Bin ${ }^{1}$, L. Boncagni $^{2}$, A. Bruschi ${ }^{1}$, S. Cirant ${ }^{1}$, G. D' $^{\prime}$ Antona $^{3}$, \\ O. D' Arcangelo ${ }^{1}$, M. Davoudi ${ }^{3}$, D. Farina ${ }^{1}$, R. Ferrero ${ }^{1}$, L. Figini ${ }^{1}$, C. Galperti ${ }^{1}$, S. Garavaglia ${ }^{1}$, \\ G. Granucci ${ }^{1}$, G. Grosso ${ }^{1}$, V. Mellera ${ }^{1}$, D. Minelli ${ }^{1}$, V. Muzzini ${ }^{1}$, A. Nardone ${ }^{1}$, S. Nowak ${ }^{1}$, G. \\ Ramogida $^{2}$, A. Simonetto ${ }^{1}$, C. Sozzi ${ }^{1}$ \\ 1 Associazione EURATOM-ENEA, IFP-CNR, Milano, Italy \\ 2 Associazione EURATOM-ENEA, C.R. Frascati, Italy \\ 3 Politecnico di Milano, Dipartimento di Energia, Milano, Italy
}

\begin{abstract}
The Electron Cyclotron (EC) fast launcher for real time control experiments has been installed on FTU and characterized to be fully integrated in a real-time MHD control module under development. The launcher scheme is based on a two module system, symmetric with respect to the equatorial plane of FTU, with a front steering concept and the launched beams are real-time controllable both in poloidal and toroidal directions. Specific design parameters, defined by the FTU MHD dynamics (typically island size and $\mathrm{q}$ profile changes), are the beam dimensions with zooming capabilities, the steering range and mirror speed with the most demanding requirement on poloidal speed of the Steering Mirror (SM) $\Delta \theta=1^{\circ}$ in $10 \mathrm{~ms}$. A set of tests has been done to verify the system performance. High power tests of the launcher have been done on a $500 \mathrm{kA}, 0.5 \cdot 10^{20} \mathrm{~m}^{-3}$ and 5.3 T plasma with $2.300 \mathrm{~kW}$ of EC power delivered to the plasma. The steering mechanism was tested under the automatic control system and showed a dynamic response in line with the requirements. Results of these tests will be presented in the paper.
\end{abstract}

\section{Introduction}

One of the missions conveyed to the new versatile EC launcher now installed on FTU [1] is to play the part of the actuator in the Real-Time (RT) control system associated to the antenna and under development [2]. The launcher was designed to inject into the plasma two independent high power mm-wave beams $(\mathrm{P}=2 \cdot 0.4 \mathrm{MW}, \mathrm{t}=0.5 \mathrm{~s}, \mathrm{f}=140 \mathrm{GHz})$ using two beam lines symmetric with respect to the equatorial plane of FTU. The dynamic of FTU MHD and the mechanical constraints fixed specific requirements to be matched by the antenna design, in particular concerning the steering mirror [3], which is realized with a small size and inertia to guarantee fast movements in the poloidal direction and with the backside covered with high emissivity layer to enhance shot to shot radiative cooling of the mirror. The front steering option gives the advantage to have a wide poloidal range, able to cover more than $80 \%$ of plasma cross section, and large toroidal injection angles as well $\left( \pm 40^{\circ}\right)$, desirable for overdense plasma heating, which is another aim of the new launcher [4]. Each beam line is also equipped with a sliding mounting able to vary the position of a shaping mirror and acting as a zoom for the launched beam and thus providing an output beam a range of possible spot sizes in the plasma center $\left(w_{\min }=19.0 \mathrm{~mm}\right.$ and $\left.w_{\max }=26.0 \mathrm{~mm}\right)$. Fig. 1 shows two views of the launcher, with the definition of the poloidal $(\alpha)$ and toroidal $(\beta)$ launching angles.

\footnotetext{
a e-mail: moro@ifp.cnr.it
}

This is an Open Access article distributed under the terms of the Creative Commons Attribution License 2.0, which permits unrestricted use, distribution, and reproduction in any medium, provided the original work is properly cited. 


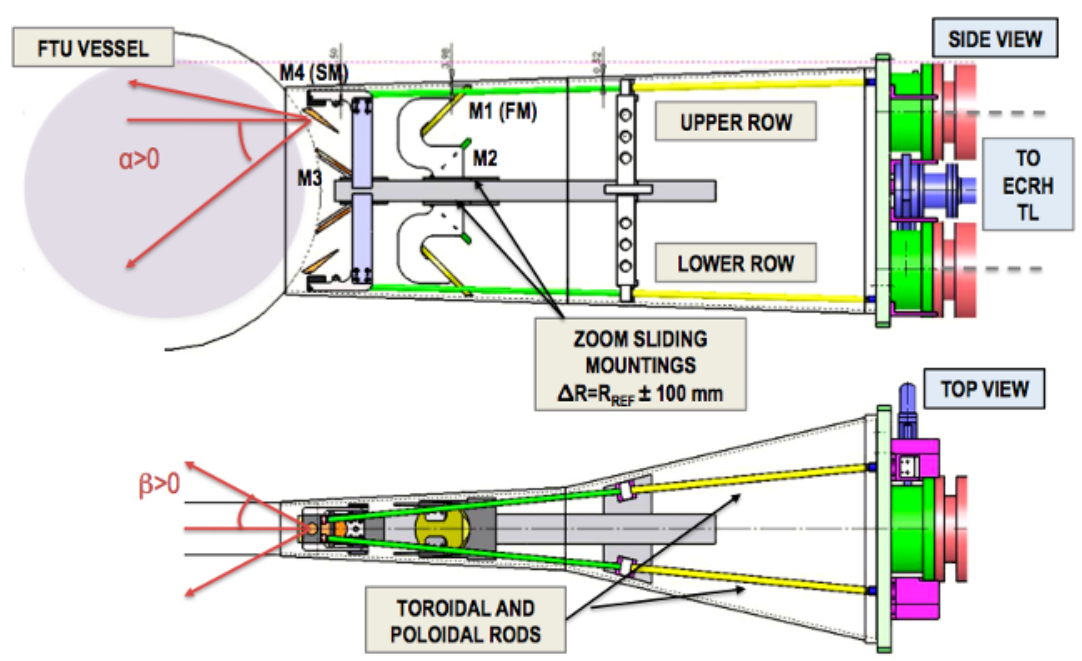

Fig. 1. Side view (top) and top view (bottom) of the EC fast launcher now installed on FTU tokamak. Poloidal $(\alpha)$ and toroidal $(\beta)$ launching angles are shown.

\section{Operational angular range characterization}

Being the antenna driving system based on rotational movements, transferred to external motors located at the rear of the port, the determination of the correspondence between the SM position (given by the poloidal and toroidal orientation angles $\theta$ and $\phi$ ) and the resulting direction of the launched beam (given by the pair $\alpha, \beta$ ), is critic in a system like this. With this in mind we firstly measured the spot position on a screen placed in front of the launcher and using a laser source. This analysis showed appreciable deviations from ideality (in particular in the case of the lower line), due to mechanical imperfections and plays present in the SM casing and driving mechanism. Prior to its installation on site, precise measurements of mechanical plays have been done tracking the laser spot position in two dimensions with an uncertainty of a tenth of $\mathrm{mm}$ by means of a Position Sensitive Detector (PSD) placed at a distance $\mathrm{D} \approx 300 \mathrm{~mm}$ in front of the antenna. As a result, the uncertainty on the launching angles introduced by mechanical plays is $\approx 0.3^{\circ}$ for both lines, corresponding to $2 \mathrm{~mm}$ at plasma axis and thus $1 / 10$ of the waist typical dimensions of the beam. Considering that SM movements are coupled, the uncertainty as a function of mirror position was also measured and 2-D pointing maps were acquired to take into account non-idealities in the manufacturing. In the mapping process the effect of hysteresis due to mechanical plays is avoided by reaching the representative points always from the same direction. Mapping was done with poloidal scans with the SM $\left(\Delta \theta=26^{\circ}\right.$, corresponding to $90 \%$ of the available range) for different toroidal positions $\left(\Delta \phi=70^{\circ}, 90 \%\right.$ of the range) allowing the experimental determination of the relations $\alpha=f(\theta, \phi)$ and $\beta=g(\theta, \phi)$, expressed in the form of a fourth degree polynomial. The functions $f^{-1}$ and $g^{-1}$ are then integrated in the control system and used to command the mirror operations. Deviations from ideality are appreciable for large injection angles, both poloidally and toroidally, as clearly visible from the data shown in Fig. 2, obtained directly measuring the laser spot positions on the target panel.

\section{Low power calibration}

As a further step of the antenna commissioning, mm-wave characterization of the launcher was done with the system plugged into FTU vessel, using a low power source inserted into ECRH transmission line and a thermopile as detector to determine and fix the reference position of the $\mathrm{SM}\left(\alpha=0^{\circ}, \beta=0^{\circ}\right)$. This procedure is necessary for overall antenna control system, as it defines the home position of the SM. 2-D radiation patterns have been measured with a dedicated automatic acquisition system that allows combined data acquisition from the detector and control of the mirror movement in the required 

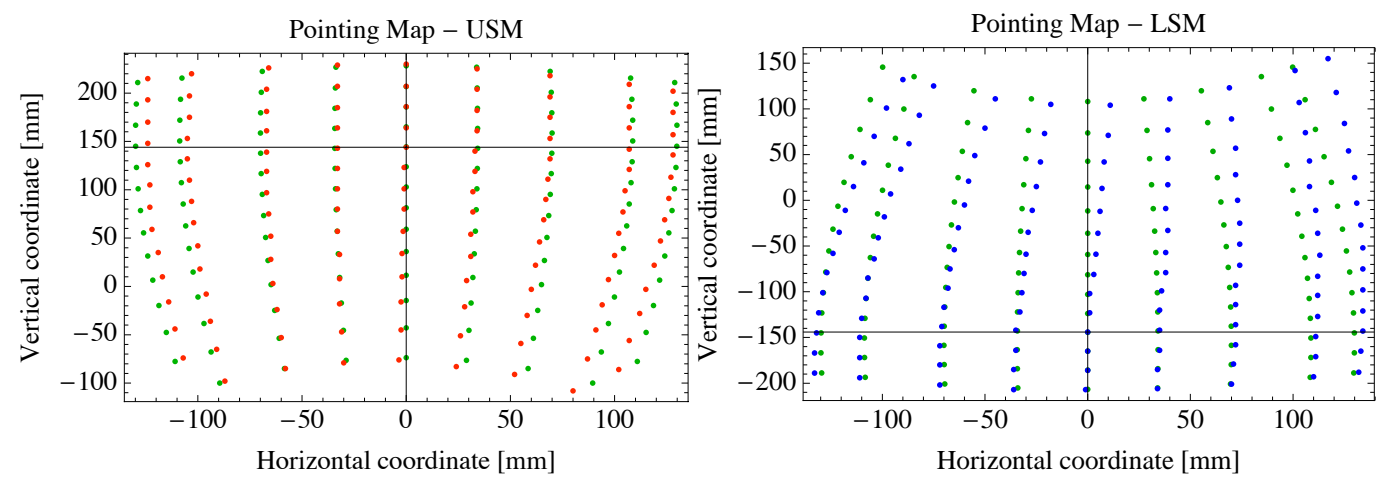

Fig. 2. Pointing maps used for optical calibration. Green dots represent ideal positions, red (blue) is used for measured upper (lower) positions. ( $\mathrm{y}=0, \mathrm{z}= \pm 144.5 \mathrm{~mm}$ ) correspond to $\alpha=0^{\circ}, \beta=0^{\circ}$. The distance from target to $\mathrm{SM}$ is $\mathrm{D} \approx 300 \mathrm{~mm}$.

angular region of the working parameters space. A few degrees misalignment of the launched beam direction was found, in the toroidal and poloidal direction. Good agreement was also found for the beam dimension as shown in Fig. 3, where both expected beam radius $\left(1 / e^{2}\right.$ of peak power $)$ and inferred 2-dimensional Gaussian profile fit have been superimposed to the contour plots. Red color is used for the theoretical spot size, green color is used for the spot size obtained from data fit. The expected beam radius at probe position ( $R=200 \mathrm{~mm}$ from vacuum vessel centre) is $13.4 \mathrm{~mm}$, and the measured values are $w_{y}=14.3 \mathrm{~mm}$ and $w_{z}=14.8 \mathrm{~mm}$ for the upper antenna, $w_{y}=w_{z}=15.7 \mathrm{~mm}$ for the lower antenna. Radiation patterns have been obtained also for different positions of the zooming system, providing values larger then expected (maximum deviation of 17\%). The larger beam radii with respect to the expected values that were found can be justified by thermopile dimensions and its sensitivity. Small deviations from previous values $(<1 \mathrm{~mm})$ appeared when similar plots were acquired with the machine cooled down at liquid nitrogen temperature and under vacuum as in operational conditions. The mechanical hysteresis of the steering mechanism was evaluated at this stage (measuring radiation patterns with different mapping techniques and comparing the position of the maximum beam intensity) and actions were taken to reduce this effect, directly acting on the cardan joints that couple rods rotation with SM movements, with a new design of these components, replaced after the first extraction of the launcher.

\section{Dynamic performances and launcher tests under plasma operations}

The control system of the SMs movement was successfully tested performing different control procedures on the antenna. Besides the antenna semi-automatic ex-vessel laser mapping and the fullyautomatic microwave mapping procedures described in Sect. 2 and Sect. 3, offline mirrors and zoom optics positioning were realized and out of boundary position recovery procedure was verified as well, in case of intervention of launcher protection system [3]. The response of the SM to a reference step movement requiring a poloidal $\Delta \theta=1^{\circ}$ in $10 \mathrm{~ms}$ was verified as well. Under plasma operations a first set of discharges was dedicated to mirrors conditioning, with increasing radio frequency power pulse length (from $t=5 \mathrm{~ms}$ to $\mathrm{t}=200 \mathrm{~ms}$ ). These shots were also used to set the best orientation of the polarizers in the ECRH transmission lines to launch a linear horizontally polarized O-mode wave into FTU. During the campaign the antenna sustained several disruption events (with plasma current $I_{P}=500$ $\mathrm{kA}$ and SM moving and also $I_{P}=500 \mathrm{kA}$ and $I_{P}=1.1 \mathrm{MA}$ with ECRH system excluded). No critical failures or damages in the system were found when the antenna was extracted at the end of the first experimental campaign, except for the deterioration of an interface plastic component that prevented the system to sustain longer gyrotron pulses unless a modulation was used.

In order to better evaluate the dynamic performances of mirror control system, pre-programmed reference mirrors trajectories have been also realized during plasma experiments and Fig. 4 shows 

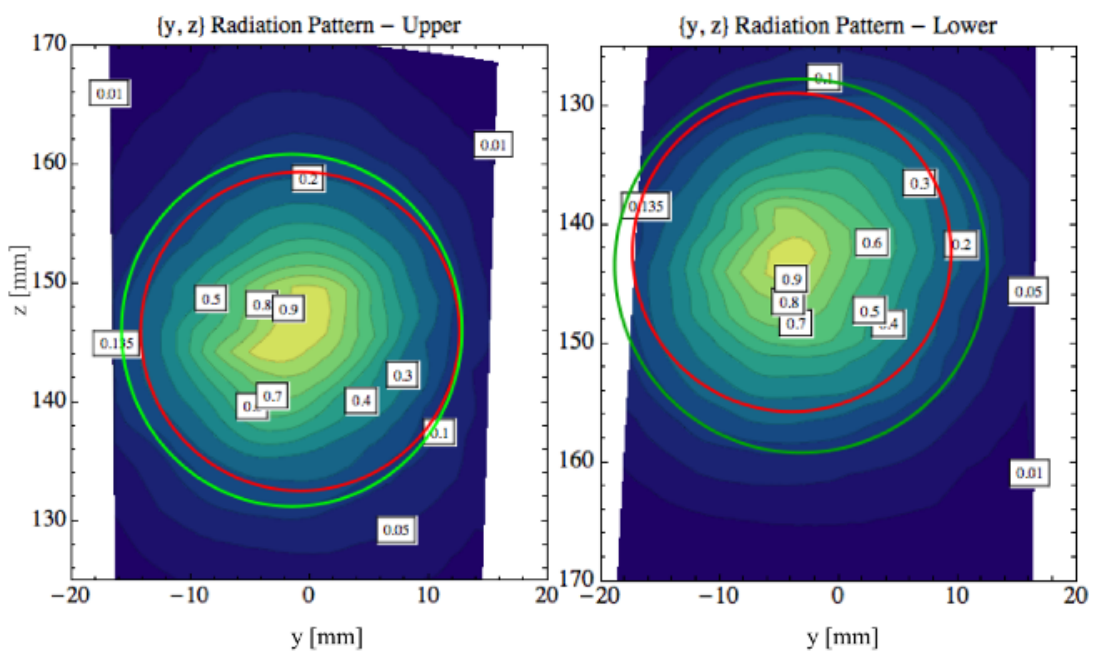

Fig. 3. Contour plot of the measured normalized radiation pattern intensity to determine the SM reference position for upper (left) and lower (right) antenna respectively. Contour level label 0.135 corresponds to $1 / e^{2}$ of the peak power, which is used to define the beam radius.

acquired SM trajectory (for upper SM) during FTU pulse \#34888 in which a poloidal $14^{\circ}$ scan in $0.2 \mathrm{~s}$ was set up $\left(\Delta \alpha=\left(28^{\circ}, 14^{\circ}\right)\right.$ and $\left.\beta=0^{\circ}\right)$. This is realized with mirror movements $\Delta \theta=7.5^{\circ}$ and $\Delta \phi=0.5^{\circ}$. The maximum discrepancy between the poloidal reference and the real angular position is $0.3^{\circ}$, while for the toroidal position is $0.06^{\circ}$, much lower in this case being the error itself proportional to the velocity. The same test (with similar results) was performed for the lower antenna as well.

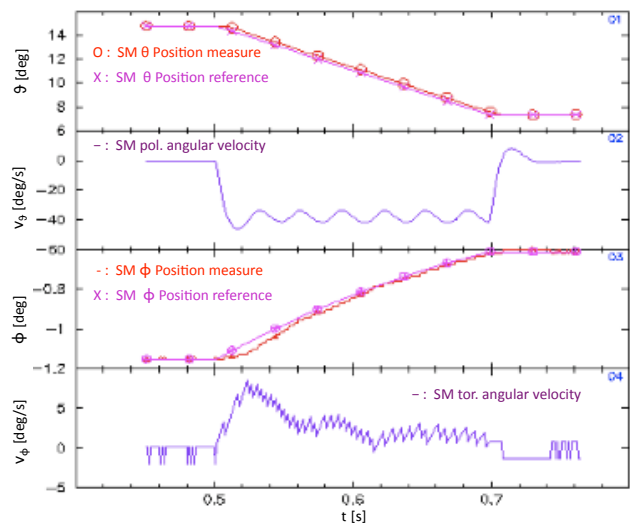

Fig. 4. Poloidal scan realized during plasma discharge \#34888. A selection of SM parameters monitored is shown: angular position, reference and velocity.

The EC power deposition location during SM movements can be inferred and followed with crosscorrelations lock-in techniques between ECE fluctuations and ECH power monitor signals (techniques used for control purposes, as described in [5]). Results provided with off-line beam tracing code GRAY [6] combined with ODIN equilibrium code [7] have been compared to the ECE-ECH cross-correlations and to calculations coming from real time ray-tracing [8] as well (FTU pulse \#34905). At different times of a plasma discharge $(\mathrm{t}=0.82,0.86,0.9,0.94$ and $0.98 \mathrm{~s})$ and during a poloidal scan of the lower mirror $\Delta \alpha=\left(-28^{\circ},-14^{\circ}\right)$ the deposition radius $\mathrm{R}$ in the equatorial plane is provided by the three previously mentioned tools. Results are summarized in Fig. 5 showing good agreement, being 
the maximum discrepancy measured of the order of $2 \mathrm{~cm}$, which is lower than adjacent ECE channels distance and comparable to the expected beam radius. A limited number of plasma discharges were dedicated to the test of the zooming feature, realized positioning the focusing mirror of the internal optics unit close to the two extreme positions of the available range $( \pm 8 \mathrm{~cm}$ with respect to its nominal position). From the mechanical point of view no apparent problems emerged but not appreciable effects of the launched beam dimensions on the plasma were found (modulated EC pulses with the SM pointing moving from plasma centre to plasma mid-radius). Further analysis is thus necessary with additional experiments and a more favourable deposition location, with the beam axis tangential to the plasma flux surfaces, corresponding to a launch with $\alpha \approx 0^{\circ}$.

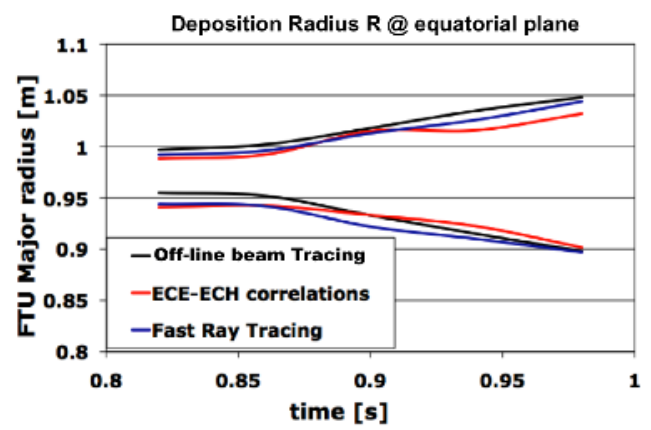

Fig. 5. Deposition radius R determination in pulse \#34905 using different tools: beam tracing code GRAY combined with ODIN equilibrium code (black), ECE-ECH cross-correlations (red) and real time ray-tracing (blue). ECE-ECH cross-correlations and real time ray-tracing are key elements of the real-time MHD control module.

Scheduled plasma discharges also included experiments to test the toroidal steering. The dynamic response of the steering mirror during FTU pulse \#34883 is shown in Fig. 6, where pre-programmed and effective trajectories $\theta(t)$ and $\phi(t)$ for the upper SM are presented. Different phases of the overall experiment are indicated: after a slow approach to the chosen initial orientation $\theta_{0}=12.7^{\circ}$ and $\phi_{0}=$ $40.2^{\circ}$ (corresponding to $\alpha_{0}=28^{\circ}$ and $\beta_{0}=-20^{\circ}$ ), a fast steering is realized to reach in $0.2 \mathrm{~s}$ the position $\theta=11.2^{\circ}$ and $\phi=-1.2^{\circ}$ (corresponding to $\alpha=28^{\circ}$ and $\beta=0^{\circ}$ ). Finally, the $\mathrm{SM}$ is slowly moved to its home position again. This is the general scheme defined to perform a pre-programmed trajectory of the SM, and will also be used in the real time MHD control system [9]. Note that in this specific case the toroidal speed commanded (and reached) is $\approx 2.5$ times greater than the required value of $10^{\circ}$ in $10 \mathrm{~ms}$.

Additional shots (\#34906 and \#34907) were dedicated to variable toroidal injection angle (overall ranging from $\beta=-20^{\circ}$ to $\beta=20^{\circ}$ ), and observation of its effects on the stray radiation, which is detected by a set of sniffer probes located in specific toroidal positions in FTU vessel. This is a preliminary step, envisioning for the antenna experiments of polarization control as well. When the real launch geometry, launched polarization (a linear horizontal O-mode in our case) and plasma parameters are included, an asymmetry in the ratio of coupled over lost power between injected and plasma waves is expected as $\beta$ changes. The asymmetry in $\beta$ is only qualitatively found as shown in Fig. 7. Further analysis will be done in the future campaign on this specific issue, with additional modeling and experiments.

\section{Conclusions}

Real time controlled injection of EC waves for experiments on FTU is being developed. As a part of this project a new antenna is now installed and many features have been characterized. In particular the issues considered to be crucial for MHD real time control experiment, which is one of the principal missions of the antenna, have been tackled and here presented. A series of test were thus realized showing good performances of the system. Optical alignment and low power measurements showed 


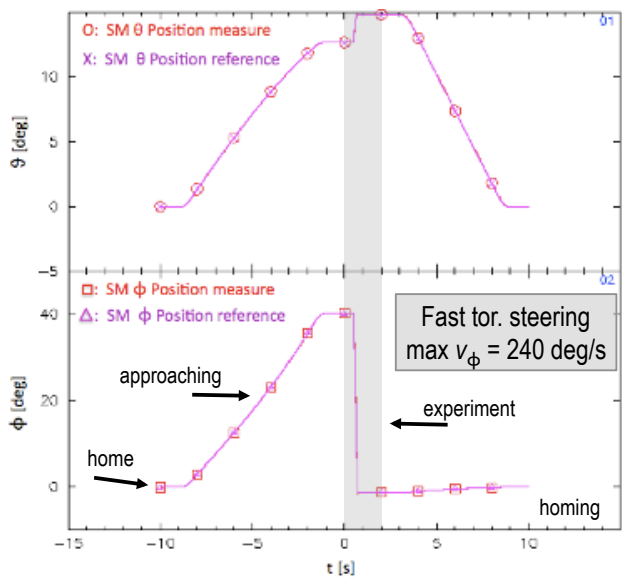

Fig. 6. Toroidal scan realized during plasma discharge \#34883. The different phases of the pre-programmed trajectory for the steering mirror are shown: starting from the home position, a slow approach to the initial value of the scan follows. Fast movement of the mirror is then realized (shaded region) and finally the mirror is slowly brought to home position again.

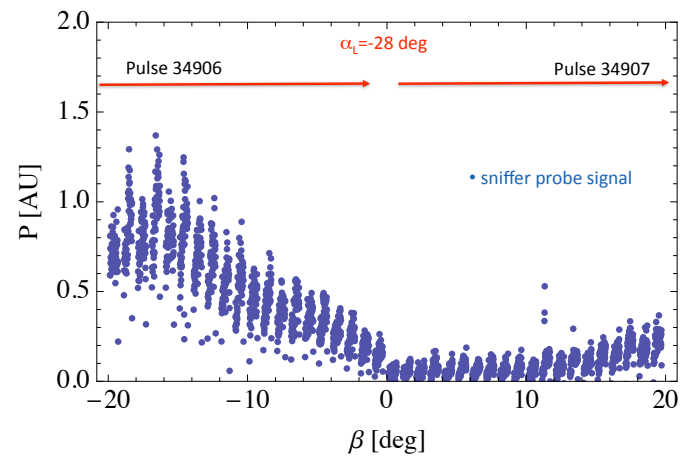

Fig. 7. Stray radiation amount as collected by one of the sniffer probes available during toroidal steering movement in the range $\beta=\left[-20^{\circ}, 20^{\circ}\right]$ realized with pulses \#34906 and \#34907.

that the steering mirror (based on a front steering concept), is able to prompt react and inject EC power when and where the control algorithm in parallel developed will require such action. The dynamic of the response is in line with the designed specifications as well. The technical problems noticed (none of them responsible of major failures or damages anyway) have been tackled and solved and preliminary data analysis of high power effects on plasma helped to identify required actions to be taken in the near future to fully finalize the commissioning of the EC launcher.

\section{References}

1. A. Bruschi et al., Fusion Sci. Technol., 55, 94-107 (2009).

2. C. Sozzi et al., This Conference.

3. A. Moro et al., Fusion Engineering and Design, 86, 942-946 (2011).

4. W. Bin et al., Fusion Engineering and Design, 84, 451-456 (2009).

5. E. Alessi et al., This Conference.

6. D. Farina, Fusion Science \& Technology, 52, 154-160 (2007).

7. F. Alladio et al., Nuclear Fusion 26, 1143 (1986).

8. S. Nowak et al., Proc. of EPS $38^{\text {th }}$ Conf. on Plasma Physics, France, 2011, P4.085.

9. C. Galpert et al., This Conference. 